\title{
References
}

Afzelius B A 1995 Situs inversus and ciliary abnormalities. What is the connection?; Int. J. Dev. Biol. 39 839-844

Brown N and Wolpert L 1990 The development of handedness in left/right asymmetry; Development 109 1-7

Collignon J, Varlet I and Robertson E J 1996 Relationship between asymmetric nodal expression and the direction of embryonic turning; Nature (London) 381 155-158

Levin M 1997 Left-right asymmetry in vertebrate embryogenesis; Bioessays 19 287-296

Marszalek J R, Ruiz-Lozano P, Roberts E, Chien K R and Goldstein L S 1999 Situs inversus and embryonic ciliary morphogenesis defects in mouse mutants lacking the KIF3A subunit of kinesin-II; Proc. Natl. Acad. Sci. USA 96 5043-5048

Nonaka S, Tanaka Y, Okada Y, Takeda S, Harada A, Kanai Y, Kido M and Hirokawa N 1998 Randomization of left-right asymmetry due to loss of nodal cilia generating leftward flow of extra embryonic fluid in mice lacking KIF3B motor protein; Cell 95 829-837

Sampath K, Cheng A M, Frisch A and Wright C V 1997 Functional differences among Xenopus nodal-related genes in left-right axis determination; Development 124 3293-3302

Supp D M, Witte D P, Potter S S and Brueckner M 1997 Mutation of anaxonemal dynein affects left-right asymmetry in inversus viscerum mice; Nature (London) 389(6654) 963-966

Takeda S, Yonekawa Y, Tanaka Y, Okada Y, Nonaka S and Hirokawa N 1999 Left-right asymmetry and kinesin superfamily protein KIF3A: new insights in determination of laterality and mesoderm induction by kif3 $\mathrm{A}^{-/-}$mice analysis; J. Cell Biol. 145 $825-836$

Krishanu Ray

Department of Biological Sciences,

Tata Institute of Fundamental Research, Homi Bhabha Road,

Mumabi 400 005, India

(Email,krishanu@tifr.res.in)

\section{Ploidy influences gene expression in yeast}

Genomes are usually regarded as static, changing only on the leisurely time-scale of evolution. This assumption clearly overlooks the changes in ploidy that cells in an organism undergo during various stages of growth and development. A mitotic cell doubles its ploidy during DNA synthesis and 
restores it subsequently at cell division. Polyploid cell types such as megakaryocytes (16n to 64n) or hepatocytes (2n to 8n) are commonly found during normal differentiation. Tumour cells have aberrant cell-cycle controls leading to an altered ploidy status. Further, deviation from the common theme of a haploid/diploid genomic constitution is widespread in the plant kingdom.

Do changes in the ploidy of a cell influence gene expression? Halving or doubling the total size of the genome would leave relative gene dosages unaffected; so can one expect patterns and relative levels of gene expression to remain identical? In the special case of reduced ploidy the answer is clearly no, because egg and sperm cells (both haploid) are highly specialised cell types. In this sense they are comparable to other differentiated cells that have their own characteristic patterns of gene expression. Because of the lack of isogenic cell types that vary only in their ploidy, a general answer to the question has hitherto eluded us.

In a recent report Gerald Fink's group presents the first convincing experimental evidence in support of the existence of a ploidy-driven mechanism of gene regulation (Galitski et al 1999). Elucidation of the precise mechanism underlying this novel regulatory phenomenon requires further experimentation. However, the knowledge that it exists and can be exploited by living systems to fine-tune gene expression adds a new dimension to our understanding of gene regulation.

Galitski et al (1999) chose to work with the budding yeast Saccharomyces cerevisiae because of the ease with which it can be manipulated and our detailed knowledge of gene expression in S. cerevisiae (extending over more or less the entire genome). By artificially inducing mating-type switching followed by successive matings, they created a ploidy series ( $\mathrm{n}$, $2 n, 3 n$ and $4 n$ ) beginning with each of the yeast cell types a, $\alpha$ (both haploids) and a $\alpha$ (diploid). In what must be the most definitive demonstration of the power and utility of microarray-based expression pattern analysis to date, Galitski et al identified 17 ploidy-regulated genes. These genes showed a monotonically increasing or decreasing level of expression (with increase in ploidy) between different members of an isogenic ploidy series. Interestingly, they were also able to demonstrate a link between the expression patterns of some of these genes and altered morphology and/or behaviour in polyploid cells. A good example of this is the ploidy-repressed CLN1 gene, a G1 cyclin. Cells with greater genome content have cell sizes that are significantly larger than normal. Also, it is well established that lowered expression of G1 cyclins causes cells to enter the cell cycle at a larger size. The demonstration that CLN1 gets repressed with increase in ploidy provides a direct link between polyploidy and cell size. There are several other examples supporting the existence of ploidy-dependent gene expression. The challenge now is to explain how the cell senses a doubling or tripling of its genome and relays this message to the transcription machinery so as to cause the repression of certain genes and the turning on of others.

\section{Reference}

Galitski T, Saldanha A J, Styles C A, Lander E S and Fink G R 1999 Ploidy regulation of gene expression; Science 285 251-254

Abhilasha Gulati

Department of Molecular Reproduction, Development and Genetics, Indian Institute of Science, Bangalore 560 012, India (Email,abhi@serc.iisc.ernet.in) 